\title{
Redesigning Undergraduate Engineering Education at MIT - the New Engi- neering Education Transformation (NEET) initiative
}

\section{Prof. Edward F. Crawley, Massachusetts Institute of Technology}

Professor Ed Crawley is the Ford Professor of Engineering at MIT, a member of the National Academy of Engineering, and a recipient of the Bernard M. Gordon Prize for engineering education of the NAE. $\mathrm{He}$ is the Founding President of the Skolkovo Institute of Science and Technology (Skoltech) and. the Co-Director of NEET at MIT.

\section{Prof. Anette "Peko" Hosoi, Massachusetts Institute of Technology}

Anette (Peko) Hosoi is Associate Dean of Engineering and the Neil and Jane Pappalardo Professor of Mechanical Engineering, at MIT. She received her PhD in Physics from the University of Chicago and went on to become an NSF Postdoctoral Fellow in the MIT Department of Mathematics and at the Courant Institute, NYU. She is a leader in the study of the hydrodynamics of thin fluid films and in the nonlinear physical interaction of viscous fluids and deformable interfaces. Her work spans multiple disciplines including physics, biology and applied mathematics, and is being used, in collaboration with Schlumberger-Doll Research, Bluefin Robotics, and Boston Dynamics to guide the engineering design of robotic crawlers and other mechanisms.

Prof. Hosoi is an exceptional, innovative teacher and an inspiring mentor for women in engineering. She was awarded the Bose Award for Excellence in Teaching, and a MacVicar Fellowship.She is a recipient of the 3M Innovation Award and has held the Doherty Chair in Ocean Utilization at MIT. She is a Radcliffe Institute Fellow and a Fellow of the American Physical Society. Her research interests include fluid mechanics, bioinspired design and locomotion, with a focus on optimization ofcrawling gastropods, digging bivalves, swimming microorganisms and soft robotics. Prof. Hosoi is also an avid mountain biker and her passion for sports has led her to create MIT Sports Lab, a program that is designed to build an interconnected community of faculty, students, industry partners, alums and athletes who are dedicated to applying their technical expertise to advance the state-of-the-art in sports.

\section{Dr. Amitava "Babi" Mitra, Massachusetts Institute of Technology}

Dr. Amitava "Babi" Mitra is Executive Director, New Engineering Education Transformation (NEET), MIT. He was the founder-Dean, School of Engineering and Technology, BML Munjal University, Gurgaon, India and the founder-President and Vice-Chancellor, Vivekananda Global University, Jaipur, India. Mitra is passionate about evangelizing and implementing an educational vision that he believes in; he enjoys formulating, designing and planning its implementation, and then taking it through to fruition. Mitra was Executive Director, Academic Media Production Services (AMPS), MIT; Senior Vice-President, Knowledge Solutions Business, NIIT (USA), Inc.; the first Chief, Distance Learning Programs Unit, BITS, Pilani, India, and; founder-member, Council of Governors, Pan-Himalayan Grassroots Development Foundation, Kumaon, India. He has served on the NERCOMP Board of Trustees, USA, was a founder-Board member, Sakai Project Board, USA and co-chaired the Advisory Board, Royal Roads University, Victoria, Canada. Mitra participated in the formulation of the Government of India's National Policy on Education 1986; this led to his being one of the authors of a book titled, "Challenge and Response - Towards a New Education Policy \& Beyond", by C.R. Mitra \& Associates, Wiley Eastern Limited, India, 1986. He studied at St. Columba's School, New Delhi, a National Science Talent Scholar, on the all-India merit list of the Indian Institute of Technology (IIT) Joint Entrance Examinations and earned a Ph.D. in chemical engineering from BITS, Pilani, India. Mitra was born in New York City and grew up in India. He loves food, rock and roll music, the intersects across people and technology, growing up with his children and playing squash. 


\title{
Redesigning Undergraduate Engineering Education at MIT --- The New Engineering Education Transformation (NEET) initiative
}

\begin{abstract}
MIT is reimagining and redesigning its undergraduate engineering education through the New Engineering Education Transformation (NEET) initiative, which is based on the following four principles that are in consonance with student and societal needs, faculty values and MIT's values and culture:

- $\quad$ Prepare our students to develop the new machines and systems that they will build in the middle of the 21 st century to address societal needs.

- Help our students to be makers, discoverers or on the spectrum.

- $\quad$ Build our education around the way our students best learn.

- $\quad$ Teach our students the NEET Ways of Thinking --- how to think, and how to learn more effectively by themselves.
\end{abstract}

NEET is a new cross-departmental project-centric academic program with formalized collaboration across majors that was initiated in September 2016. Students will get a degree from the department they are majoring in plus a NEET Certificate in the cross-disciplinary thread they have opted for.

We have identified a framework of eleven NEET Ways of Thinking; this framework formed the basis for getting inputs and gathering evidence from a range of stakeholders, including thought leaders, industry, alumni, students and faculty. An independent consultant was commissioned to conduct a global undergraduate engineering education survey. We will share the findings from the evidence gathered and elaborate on the three inventions of NEET that emerged from looking at the evidence: the NEET Ways of Thinking; the project-centric curricular construct, and; the concept of threads. The main organizing armature of the curriculum is now a sequence of projects inspired by the new machines. Students choose a sequence of explicitly interdepartmental projects, while fundamentals continue to be learned in departmentally offered subjects. Threads are pathways for inter-disciplinary engineering education that cut across disciplines and departments. Threads could be envisioned in areas such as the Internet of Things, autonomous vehicles and systems, smart cities and urban infrastructure, and, large data analysis systems.

Engineering education worldwide is struggling with the widening gap between theory and practice. Employers increasingly believe that their needs are not being met. It is not that the education system has broken down; it's just that what it 
delivers is increasingly obsolete. NEET aims to make engineering education more relevant.

NEET (see neet.mit.edu) launched two pilot threads in Fall 2017, Autonomous Machines (covering aeronautics \& astronautics, mechanical engineering and electrical engineering \& computer science) and Living Machines (covering biological engineering, mechanical engineering and chemical engineering, but also open to any MIT undergraduate pursuing a technical degree program), and will be launching two new pilot threads in Fall 2018, Advanced Materials Machines (covering materials science and engineering and mechanical engineering) and Low Carbon Energy Systems (covering nuclear science and engineering, civil and environmental engineering and mechanical engineering). We will describe the process of building the community of students and faculty and developing curricula and projects for the threads, and share the learnings from the pilots, proposed governance structures, measures of success and opportunities on the horizon.

Though it is far too early to come to meaningful conclusions for the medium to longer term, the initial trends are encouraging. Over $5 \%$ of the sophomore engineering population has voluntarily opted for NEET, a significantly larger number than the number of students that have typically tended to opt for many new academic programs in the past. The student newspaper and a student blogger have also covered NEET favorably in the short space of eight months. 


\section{The Context}

Chuck Vest who served as president of MIT and of the National Academy of Engineering has said that much of what we view as engineering fundamentals was shaped by what is commonly termed "engineering science". This approach evolved through World War II and continued after that since scientists were increasingly coming to the forefront as inventors. The pendulum swung a bit too far from practical engineering, and it is now time to find the right balance. According to Vest, "Students must learn how to conceive, design, implement and operate (CDIO) complex engineering systems of appropriate complexity."1

The last two-three decades have seen exponentially rapid development of technology. Engineering practice and education have not kept pace. Sheppard et $\mathrm{al}^{2}$ explore three central questions, the most important of which is, "Is the engineering curriculum organized and delivered in ways that align with what engineers must know and be able to do, now and in the future?"2 The overwhelming response is that the curriculum does not address the machines and systems of the future.

Bok $^{3}$ has argued that teaching students how to think and how to think critically is the primary aim of undergraduate education, rather than accretion of credit units and content. Yet the vast majority of undergraduate programs do not recognize this issue and those few that do are at somewhat of a loss as to how to implement this.

Engineering education research has made giant strides in the past twenty years or so and it is clear that "a robust research base is required to inform future engineering practice" . Yet we see few applications of this in creating innovative curricula and approaches to teaching and learning.

University teaching is probably the only profession where novice practitioners, i.e., new faculty with fresh $\mathrm{PhD}$ 's are expected to start off as experts without any kind of systematic training. Rapid advances have been made in the "science of learning" and taking an evidence-based approach to college teaching can help create more effective teachers ${ }^{5}$. Yet faculty are rarely trained on how to deal with changes in curricular approach and curriculum or when new pedagogy is introduced.

The institutional prescriptions for improvements in the system of education have largely been suggestions for better management. There are proposals that "...we need to rethink and, of consequence, restructure what we do"6. They offer "strategic ways for the academy to act rather than merely produce a philosophical tract arguing that times are bad, change is needed, and institutions must find a "vision."

It is believed that the late management guru Peter Drucker famously said:" Culture eats strategy for breakfast." Fundamentally, any change including changes in curricula and educational approach requires a change in culture that builds on the values of the institution. 


\section{What is NEET's Goal?}

NEET (New Engineering Education Transformation) was chartered by the Dean, School of Engineering, MIT in the summer of 2016 and initiated in September 2016. The aim is to reimagine and rethink engineering education - what students learn and how students learn - in a fundamental way across the school.

NEET (New Engineering Education Transformation) is an interdepartmental project-centric academic program with formalized collaboration across departments. Starting from their sophomore year, students choose a sequence of explicitly interdepartmental projects in their sophomore, junior and senior years, while fundamentals continue to be learned in departmentally offered subjects. Students are coached in personal and interpersonal skills and are challenged to develop their ability to learn by themselves. This initiative aims to educate young engineers to build the new machines that will address societal needs, by preparing them to work as entrepreneurs/innovators and discoverers, and by instilling the NEET Ways of Thinking.

Students will get a degree from the department they are majoring in, plus a NEET Certificate in the cross-disciplinary thread they have opted for.

\section{The Strategy of NEET}

NEET is a student-focused endeavor. The charter from the Dean of Engineering focuses on the following four principles that capture student and societal needs, faculty values and the MIT culture. These principles are:

- Our education should focus on preparing our students to develop the new machines and systems that they will build in the middle of the $21^{\text {st }}$ century to address societal needs.

- We should help our students to prepare themselves to be makers, discoverers or along this spectrum, and we should teach engineering fundamentals as a foundation for careers both in research and in practice.

- We should build our education around the way our students best learn, engaging them in their learning, and implementing pilots to understand the desirable balance of classroom, project and digital education.

- In view of the speed of scientific and technological development, we should teach students the NEET Ways of Thinking, how to think, and how to learn more effectively by themselves.

We should be prepared to embark on a bold change, with widespread impact at MIT and potentially elsewhere.

The values of MIT have remained unchanged since its founding. To summarize, these focus on:

- Useful knowledge. “... in industrial society, science and technology were legitimate foundations for higher knowledge..."7

- Societal responsibility. "... to apply the fruits of scientific discovery to the satisfaction of human wants"7

- Learning by doing. “... converting personal experience into knowledge.”7 
- Education as preparation for life. "... provide students with an education that better prepares engineers to function as professionals..."8

- The value of fundamentals. "...education should be based on the fundamental principles..."8

The key principle is an orientation towards new machines and systems. We use the term "machines" generally to describe all of the things that engineers build, including infrastructural, informational, molecular, mechanical and biological constructs. What are the new machines? Simply put, these are the things that we must prepare our graduating students to build in the middle of their careers, 20 to 30 years from now, to address societal needs. Here is an example of what we have termed as "old machine" and "new machine".

\section{Flying Machines}

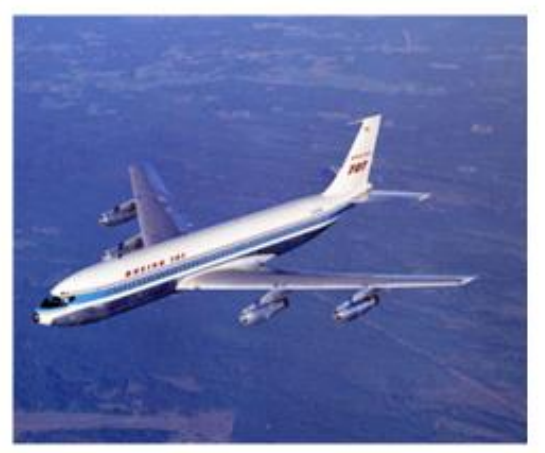

1950's “Old Machines"

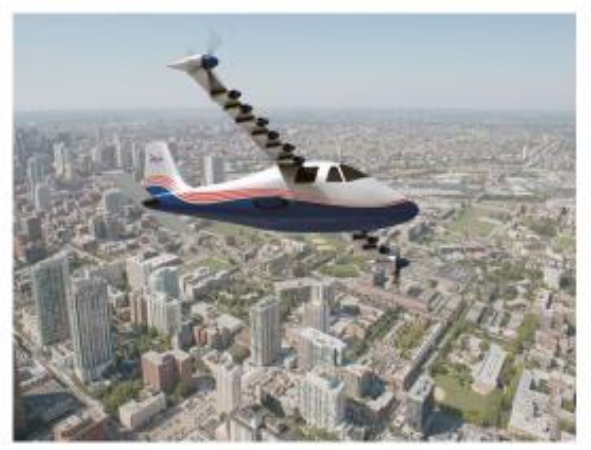

Today's “New Machines"

The airplane on the left is a 1950's era Boeing 707; it is designed based on aerodynamics. The flying machine on the right is NASA's X-57 Maxwell, an aircraft that looks like the one on the left but is being developed based on electric propulsion using lithium-ion batteries; they estimate that it will reduce fuel use by a factor of five, be emission-free, have $40 \%$ lower operational costs and be much quieter than conventional aircraft. Unfortunately, the aeronautical engineering curriculum at most universities is oriented towards getting students to design the "old machine". Designing the "new machine" on the right would at best be covered in one course. We must work energetically to make this shift in order to overcome academic inertia, the conservative influences of accreditation and professional societies, and the hiring practices of major companies.

The second principle of NEET is that we should help our students prepare themselves for careers on a spectrum from makers to discoverers. Makers are those who will have careers as entrepreneurs and innovators - those who will make inventions and actually conceive, design, implement and operate systems and products that deliver value. Discoverers are those who will 
have careers as researchers and in $\mathrm{R} \& \mathrm{D}$--- those who will create new knowledge and increase our understanding of phenomena.

Another NEET principle is that we should build our education around the way our students best learn, engaging them in their learning and self-learning. We should increase the use of the approaches proven to work, including more active engagement of students in the classroom, more project exposure, and creative uses of digital learning and professional experiences.

\section{Progress and Activities during 2016-17}

NEET (see neet.mit.edu) was initiated in September 2016.

The first stage focused on 'Formulating the NEET Approach'. This included stakeholder analysis, extensive discussions in the weekly core NEET faculty committee meetings, consultation with individual members of the extended NEET faculty committee, and deliberation about high-level goals and master architectures in a workshop with students, industry, alumni and faculty that was held in September 2016. The concept of 'New Machines and Systems' emerged during this stage. This led to in-depth conversations in October 2016 with the extended NEET faculty committee at a workshop titled 'Gathering the Evidence' as to what kind of evidence needed to be gathered, from whom, and what the modalities could be. The two artifacts that emerged from this stage were a synopsis of the NEET initiative, and the NEET Ways of Thinking (see Table 1).

The second stage focused on 'Gathering the Evidence', which involved getting inputs from a range of stakeholders, including around six thought leaders, forty senior managers from industry, around forty alumni/ae, over forty-five students, around thirty-two faculty and about eight institutional leaders ${ }^{9}$. The modalities and procedures for gathering inputs included telephonic interviews, online surveys, TED Talks, YouTube videos, one-on-one discussions, workshops, focus groups and Town Hall meetings. Sugata Mitra, the education scientist who created the 'Hole in the Wall' learning project opined in his 2013 Prize Winning TED Talk ${ }^{10}$ that our current system of educational mass production is working but is obsolete. It is this conclusion --- that the system isn't broken but needs to become more relevant --- that has guided NEET's approach.

We commissioned Dr. Ruth Graham an independent educational consultant who focuses on higher education reform to conduct a global undergraduate engineering education survey ${ }^{11}$ to identify institutional leaders and innovators, and innovative educational constructs. The key findings from this study were that emerging institutional leaders have benefited from strong and visionary academic leadership, a faculty culture of educational innovation, and new tools that support educational exploration and student assessment. There were two trends that emerged. The first is a tilting global axis to Asia and the South, benefiting from strategic government and private investment as incubator for economic development. The second is distinctive studentcentered curricular experiences with an integrated educational approach that are being implemented at scale. 
The third stage looked at 'Working out the NEET Options'. The initial findings and analysis of the evidence gathered were presented at a workshop in December 2016 on 'Evidence and Ideation' where the participants were from the core and extended NEET faculty committees. The discussions focused on whether the four principles were supported by the evidence, whether anything rose to the level of another principle, and what were the operating concepts that might be considered for a NEET-based approach to engineering education. Two of the key artifacts that emerged from this stage were an articulation of the attributes of new machines and systems, as well as the concept of threads which would coalesce areas and pathways of study that would cut across disciplines and departments. Examples of threads could be the Internet of Things, autonomy and robotics, sustainable materials and energy, medical and bio-devices, and smart cities and infrastructure. This was followed by a workshop in January 2017 on 'Working out the NEET Options' where discussion focused on operating concepts that emerged from the December 2016 workshop. The main invention of NEET, i.e., the project-centric curricular construct and scaffolding for NEET threads was an outcome of the January 2017 workshop.

Stage four, 'Working out the NEET Threads' was kicked off in February 2017. Detailed evidence gathered from stakeholders, analysis and trends were presented by core NEET committee members at a workshop held in April 2017 on 'Working out the NEET Threads' which resulted in identifying and prioritizing likely threads, identifying those that will be launched in Fall 2017, identifying other faculty members to engage, adopting a plan for work leading to curriculum review in June 2017 and organizing a cross cutting project team to develop, in cooperation with the threads, the project components, particularly focusing on common elements and resources. This workshop included nominees of members of Engineering Council, Undergraduate Academic Officers, faculty teaching subjects in the threads identified, leadership from the freshman learning communities including Concourse, the Experimental Study Group, Terrascope and the Media Arts and Sciences Freshman Program and experts from the Office of Digital Learning, the Gordon Engineering Leadership (GEL) program, the Teaching \& Learning Lab, the Registrar's Office, the MIT Libraries and other entities. The Dean of Engineering joined this workshop. The unifying project-centric construct was presented and discussed, including supporting concepts. Breakout groups examined the role of digital learning, the nature of the curriculum and the need for faculty support through an organization such as a NEET Academy. We subsequently received feedback and ideas about NEET through presentations at MIT's Academic Council, the apex body chaired by the President, and through discussions with chairs of the faculty, Deans of the various schools, Departmental Undergraduate Education Committees, the Committee on Undergraduate Performance, the Committee on the Curriculum, and, the Subcommittee on the Communication Requirement.

We launched the process of building the NEET community during this stage. A cornerstone of this process was the informal NEET faculty lunch discussions that were initiated in February 2017; the discussions have focused, for example, on hands-on experiences students go through in freshman learning communities and freshman advising seminars, on the development of personal and interpersonal skills, development of ethics, projects, project pedagogy and the development of threads. 
In April 2017 we initiated the fifth stage on 'Planning the Pilot NEET Threads'. This culminated in a two-day workshop in June 2017 where we conducted a detailed review of the status of all NEET threads under consideration and their preparedness to launch in Fall 2017 including drafts of "catalog level descriptions" of threads, learning outcomes, curricular structure of required and elective subjects, the plan for thread projects and the agreement of participating departments.

Two mini-workshops were held during May 2017 and June 2017 on 'Effective Practices in Project Classes.' They had the explicit goal of learning from faculty who have deep experience in designing and conducting project classes at MIT. NEET heard from them about defining learning outcomes for projects, best practices that they have developed or adopted, and improvements that could be made. NEET benefited from hearing about conceptual models of project classes that it should be looking at. Data was collected on the modality of projects, project durations, number of students enrolled, the number of students in a team, the number of TAs, outside resources tapped, Communication Intensive (CI) instructors, project management coordinators, other personnel involved, administrators, EHS staff, Lab Directors and budgets/costs. A key learning from these workshops as well as what we heard from students was that projects needed to be somewhat complex but also 'doable' in order for them to be worthwhile educational experiences through which students achieved specific outcomes. It was essential to structure the projects with milestones, align with lecture classes and provide continuous technical support and guidance. This has led to NEET creating a full-time budgeted project instructor role termed the Lead Laboratory Technical Instructor who in collaboration with the faculty and other teaching staff, is responsible for tactical execution and operational oversight of all the project-centric aspects of the thread. The outcomes from these mini-workshops are being aggregated to create the NEET Projects Handbook. This will help inform design of the projects that are being planned in the NEET threads during 2018-19.

To summarize, the key workshops held during the 2016-17 academic year were:

- September 2016: 'Formulating the NEET Approach'

- October 2016: 'Gathering the Evidence'

- December 2016: 'Evidence and Ideation'

- January 2017: 'Working out the NEET Options'

- April 2017: 'Working out the NEET Threads'

- May and June 2017: 'Effective Practices in Project Classes'

- June 2017: 'Planning the Pilot NEET Threads'

\section{Three Inventions of NEET}

There are three fundamental ideas that have emerged from the evidence gathered, its analysis, discussions and deliberations: what we have termed as the 'NEET Ways of Thinking'; the project-centric curricular construct, and; the concept of threads. 


\section{NEET Ways of Thinking}

At the university, students need to learn how to think, and how to learn more effectively by themselves. We have identified about eleven NEET Ways of Thinking that are cognitive approaches used by successful technical professionals. They comprise making, discovering, interpersonal skills, personal skills and attitudes, creative thinking, systems thinking, critical and metacognitive thinking, analytical thinking, computational thinking, experimental thinking and humanistic thinking (see Table 1 below).

Table 1. NEET Ways of Thinking

\begin{tabular}{|c|c|}
\hline $\begin{array}{l}\text { Ways of } \\
\text { Thinking }\end{array}$ & Description \\
\hline Making & $\begin{array}{l}\text { Innovating, by inventing and bringing about artifacts that have } \\
\text { never before been in existence: Conceiving (understanding needs } \\
\text { and technology, and creating concept), designing, implementing } \\
\text { and operating products and systems that deliver value }\end{array}$ \\
\hline Discovering & $\begin{array}{l}\text { Advancing the knowledge of our society and world by exploring, } \\
\text { identifying, and generating new learning, often by conducting } \\
\text { research that employs scientific methods and leads to new } \\
\text { fundamental discoveries and technologies }\end{array}$ \\
\hline $\begin{array}{l}\text { Interpersonal } \\
\text { Skills }\end{array}$ & $\begin{array}{l}\text { Engaging with and understanding others: communicating, } \\
\text { listening, dialog and emotional intelligence, working in and } \\
\text { leading teams, collaboration and networking, advocacy and } \\
\text { leading change }\end{array}$ \\
\hline $\begin{array}{l}\text { Personal Skills } \\
\text { and Attitudes }\end{array}$ & $\begin{array}{l}\text { Initiative, judgment and decision making; responsibility and } \\
\text { urgency; flexibility and self-confidence; acting ethically and with } \\
\text { integrity; social responsibility; dedication to lifelong learning }\end{array}$ \\
\hline $\begin{array}{l}\text { Creative } \\
\text { Thinking }\end{array}$ & $\begin{array}{l}\text { Forming something new and somehow valuable, for example by } \\
\text { focusing thought, incubating new ideas, illuminating them } \\
\text { in conscious awareness, and verifying }\end{array}$ \\
\hline $\begin{array}{l}\text { Systems } \\
\text { Thinking }\end{array}$ & $\begin{array}{l}\text { Predicting emergence of the whole by examining of inter-related } \\
\text { entities in context, in the face of complexity and ambiguity, for } \\
\text { homogeneous systems and systems that integrate multiple } \\
\text { technologies }\end{array}$ \\
\hline $\begin{array}{l}\text { Critical and } \\
\text { Metacognitive } \\
\text { Thinking }\end{array}$ & $\begin{array}{l}\text { Assessing the worth or validity of something that exists, by } \\
\text { analyzing and evaluating information gathered from observation, } \\
\text { experience or communication }\end{array}$ \\
\hline $\begin{array}{l}\text { Analytical } \\
\text { Thinking }\end{array}$ & $\begin{array}{l}\text { Working systematically and logically to break down facts and } \\
\text { resolve problems, identify causation and anticipate results, often } \\
\text { by applying theory, modeling and mathematical analysis }\end{array}$ \\
\hline
\end{tabular}




\begin{tabular}{ll}
\hline $\begin{array}{l}\text { Computational } \\
\text { Thinking }\end{array}$ & $\begin{array}{l}\text { Using computation to understand physical, biological and social } \\
\text { systems by applying the fundamental constructs of computer } \\
\text { programming (abstractions, modularity, recursion), data structures, } \\
\text { and algorithms }\end{array}$ \\
Experimental & $\begin{array}{l}\text { Conducting experiments to obtain data: selecting measurements, } \\
\text { determining procedures to validate data, formulating and testing } \\
\text { hypotheses }\end{array}$ \\
Tumaning & $\begin{array}{l}\text { Developing and exploiting a broad understanding of human } \\
\text { society, its traditions and institutions: knowledge of human } \\
\text { cultures, human systems of thought, the social, political and } \\
\text { economic frameworks of society; and modes of expression in the } \\
\text { arts }\end{array}$ \\
\hline
\end{tabular}

The NEET Ways of Thinking are the cognitive approaches and skills students need to learn and practice while still in school, so that they are better equipped with the personal effectiveness, academic and workplace competencies required once they graduate. These workplace competencies have been articulated elsewhere; one of the recent approaches is the Engineering Competency Model $^{12}$ developed by the American Association of Engineering Societies (AAES) and the U.S. Department of Labor in 2015.

\section{The NEET Project-Centric Curricular Construct}

The main invention of NEET is the project-centric curricular scheme (Figure 1). The organizing armature of the thread is now a sequence of projects inspired by the new machines. Starting in the sophomore year, students choose a sequence of explicitly interdepartmental projects, while fundamentals continue to be learned in departmentally offered classes. Students are coached in personal and interpersonal skills and are challenged to develop their ability to learn by themselves. These are supplemented by digital education, peer-to-peer learning, faculty mentoring and self-study, which stress the fundamentals. Projects would then form a basis of evaluation as well as a conduit for students to learn personal and interpersonal skills. The means of acquiring the fundamentals is less important than demonstration that the student has acquired and can apply the knowledge. In contrast, the existing class-centric curricular scheme focuses on a well-defined sequence of coursework of increasing specialization that is typically evaluated through closed-ended problem solving. Projects are viewed as supplemental, diminishing time available for the "core". It is important to note that project-centric is not the same as the wellknown project-based learning(PBL) methodology that has been pioneered by the University of Aalborg, Denmark ${ }^{13}$. In the project-centric scaffolding, the center of gravity of the curriculum shifts to the projects; PBL is a teaching method in which students gain knowledge and skills by working in a project while taking a class. 


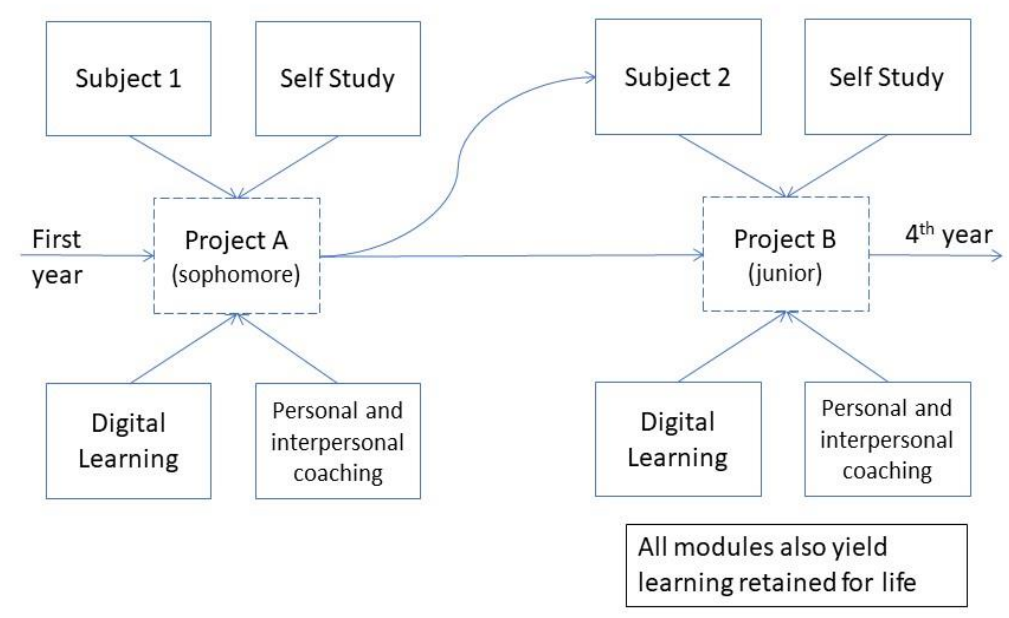

Figure 1. The NEET Project-Centric Curricular Construct

Organizing Curricula in Threads

Threads are pathways for inter-disciplinary engineering education that cut across disciplines and departments (Figure 2). Threads could be envisioned in areas such as the internet of things, autonomous vehicles and systems, smart cities and urban infrastructure, large data analysis systems, sustainable materials, and low carbon energy systems.

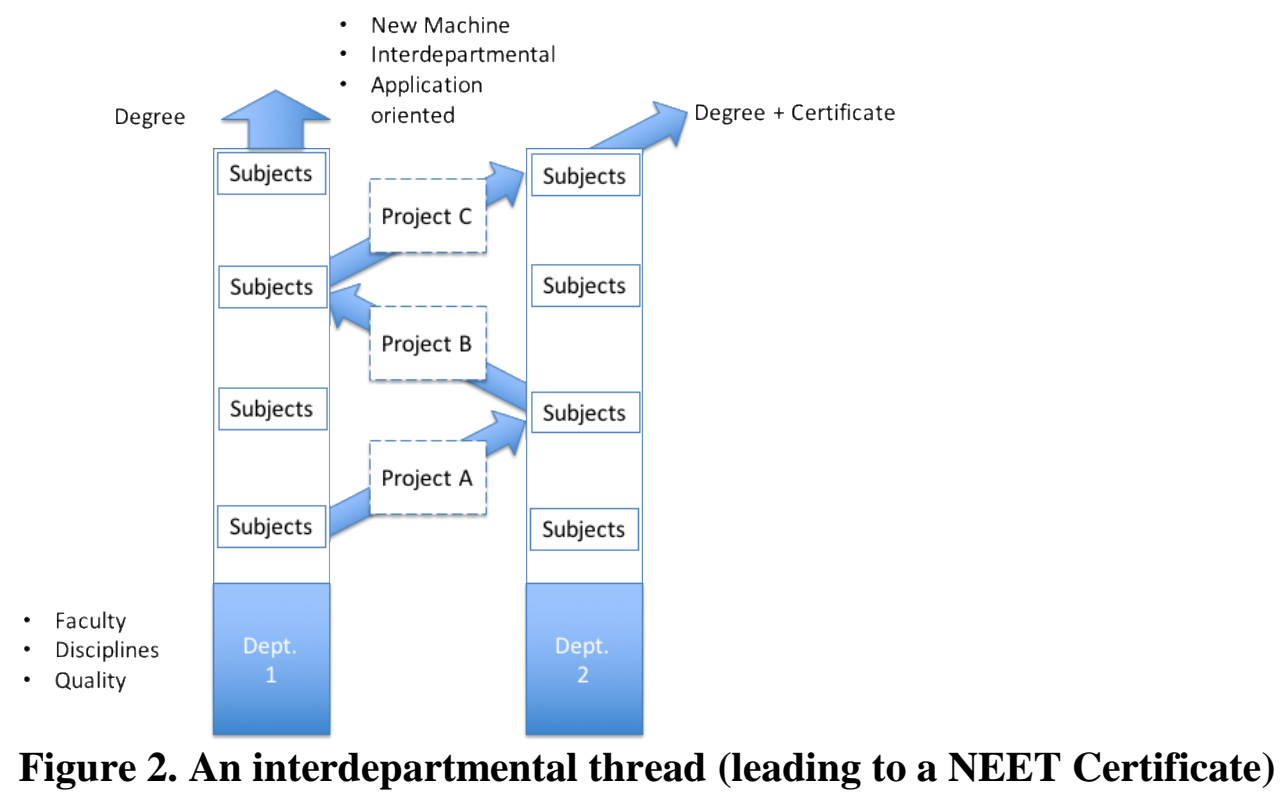

What characterizes a thread? It prepares students to participate in some new technology application (the new machines and systems); explicitly provides for learning in the NEET Ways 
of Thinking; provides a series of project-centric experiences in the sophomore, junior and senior years; has an interdepartmental nature, and; allows the option for delaying the declaration of a major from among two or three departments. During the next four pilot years, students going through the threads must be able to obtain an existing engineering degree as well as a NEET Certificate in the thread they have opted for. This last provision provides a safety net for students in case they are unable to or don't want to continue in NEET; they don't "waste" any units and graduate with a degree in their major.

A 2011 conference paper proposed that within disciplines there are critical concepts that are both transformational and troublesome for students ${ }^{14}$. By identifying and investigating these 'threshold concepts' it is possible to focus curricula. Such threshold concepts are orthogonal to threads. For example, a thread in autonomy and robotics would define a cross-departmental learning pathway integrating aspects of aeronautical engineering, mechanical engineering and computer science. Threshold concept examples include the directions in bending moment diagrams, the physical meaning of a circuit diagram, vectors, and conservation principles.

\section{Pilot Threads launched in Fall 2017}

The initial thought was to launch a couple of pilot threads in the fall of 2018. However, it was felt that significant momentum had developed in the university community and that NEET needed to 'seize the moment'. In an extraordinary show of support and organizational coherence, the MIT community decided in the spring of 2017 to launch two pilot NEET threads in Fall 2017, less than a year after initiating the project. These were: Autonomous Machines (integrating aspects of aeronautics \& astronautics, mechanical engineering and electrical engineering \& computer science) and Living Machines (integrating aspects of biological engineering, mechanical engineering and chemical engineering, but also open to any MIT undergraduate pursuing a technical degree program). For each thread, we designed a sophomore year that:

- Provides an introduction to several of the disciplines involved in the new machine.

- Incorporates a pilot section of an existing sophomore project class focused on the new machine.

- Builds student cohort unity through a special weekly NEET seminar.

- Allows participating students to take a common thread curriculum recognized by each of the participating departments as an acceptable course of study, providing them with the opportunity to essentially defer their choice of major by one or two semesters.

NEET appeals to students who may want to defer their major, work on a departmental boundary, or focus more intensely on project learning. It provides an exciting alternative to the current preponderance of interests in computer science and mechanical engineering.

NEET was oversubscribed with 56 applicants by the beginning of the fall term of 2017 . There were 43 students who signed up, 31 in Autonomous Machines and 12 in Living Machines. This represents more than $5 \%$ of the sophomore engineering class. 


\section{Operations for 2017-18 and for 2018-19}

NEET has proposed a four-year pilot program. The goal in the steady state is to offer about seven-eight threads with about 50 students in each thread, thus addressing about $50 \%$ of the undergraduate engineering students. The current year 2017-18 is the first year of the four-year pilot, and the next academic year 2018-2019 will be the second.

Two pilot threads are being implemented in 2017-18 and the proposed plan is to continue these two threads in 2018-2019, as well as possibly add two new pilot threads. The activities necessary to do this are highlighted in Figure 3 below.

The primary activities involve requesting for department-supported thread proposals from faculty, selecting the threads that will be launched, designing the sophomore year and projects, planning for the junior and senior years, marketing and recruiting students, implementing the pilot threads and obtaining feedback. There are a number of other ongoing operations. These are briefly described below:

- Classes: Our main contact in fall 2017 with the NEET students was through weekly NEET Seminars being offered in each thread. These introduced the students to the content of the thread, provided a wider exposure through presentations by industry experts and researchers, helped in building community and provided for feedback and voice of the customer. We have recruited staff to help teach the project subjects and are preparing to teach the projects in each thread in the spring.

- Program Assessment: We are working with assessment experts in the Teaching \& Learning Lab to develop a scheme to test the effectiveness of NEET and guide future program development. Data and feedback is being gathered in the process of piloting and implementing the two existing threads. This data would be analyzed, and the learning used to formally launch two new threads in Fall 2018, refine the second offering of Autonomous Machines and Living Machines and prepare for implementing pilots of other threads in Fall 2019.

- Fundraising: Efforts have been initiated to raise funds for NEET in order to strengthen medium to long-term sustainability. We have had discussions with the school's and university's Resource Development departments, have floated preliminary proposals to several potential donors and are in the process of creating a plan to raise an initial amount toward building this initiative. In the steady state, we are looking to build a corpus, the interest from which together with base funding from the school will be sufficient to operate and refine implementation of about seven-eight threads.

- Cross-School Initiatives: NEET has met with the deans of the other schools and there is considerable interest in exploring ways to offer threads that cut across schools. NEET would look to draw in other schools to support projects and the NEET Ways of Thinking and to expand the interdisciplinary nature of the threads.

- The NEET Academy: We learned from Phase 1 of the global undergraduate engineering study ${ }^{11}$ that many of the institutions identified as leaders have been thoughtful and systematic about supporting their faculty through the technical and culture changes that are called for when any new initiative is implemented. The NEET Academy is our conceptual approach to addressing how we can support our faculty through the 
development, implementation and refining of NEET. We will begin to think through its goals, scope and modality.

- Recruitment of staff: The core team is already in place. A full-time executive director staffs NEET. We have recruited the project instructors for the Living Machines and Autonomous Machines threads. We are aiming to recruit other key personnel required for the current threads and for the two new threads by July 2018, as they will need to work with the faculty on classes and projects to be offered in fall 2018 and spring 2019.

- Other ongoing activities in 2017-18 include: advising students in the threads in conjunction with departmental advisors; building the community of faculty and students for the threads; developing a common student space to help build student community and promote project work; communication including revision of the NEET web-site, and; presentations at universities, conferences and other forums.

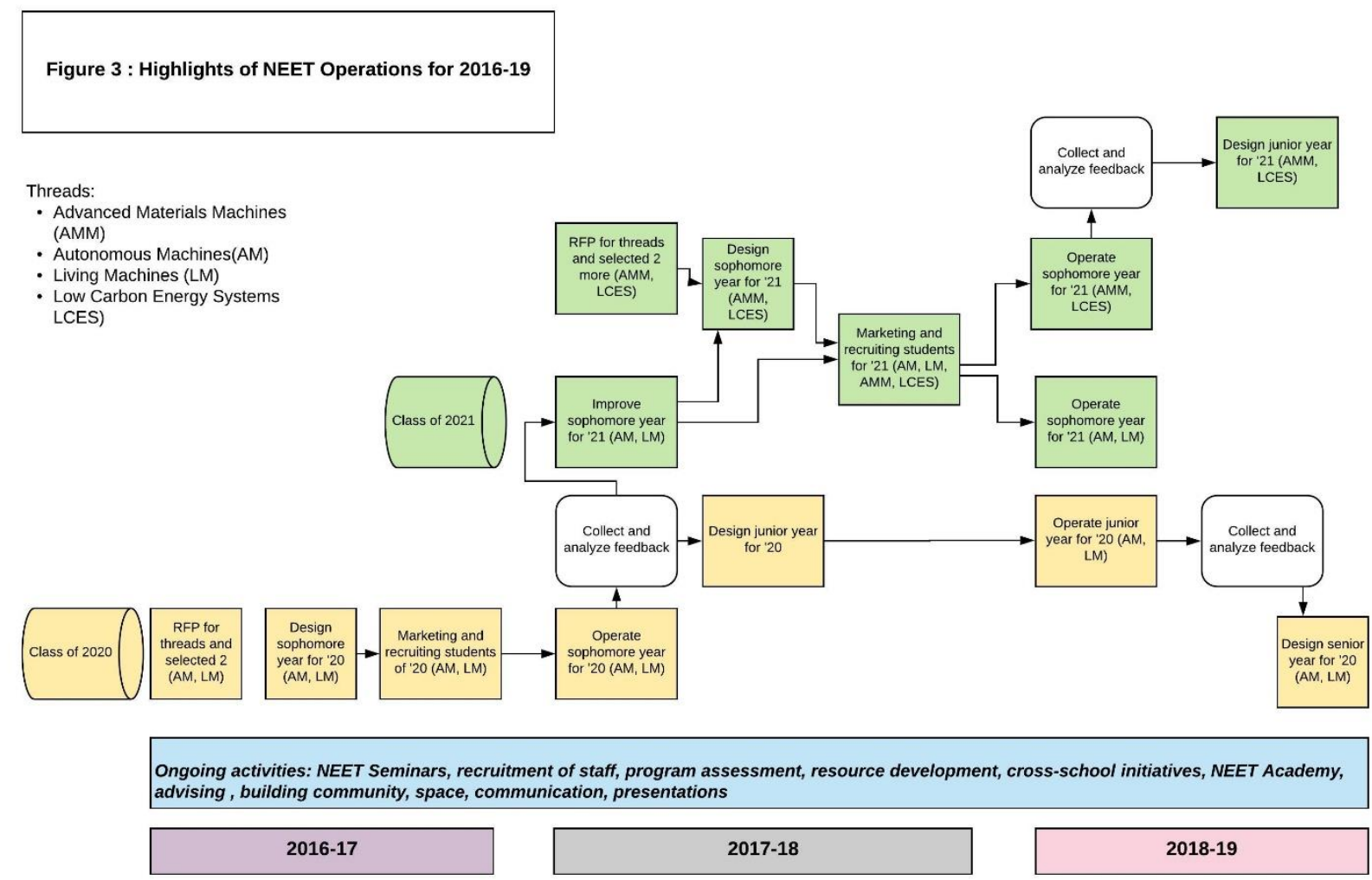

In October 2017 we started the process of launching new threads by sending out a request for thread proposals (RFP) to be launched in Fall 2018. These included criteria for the new threads and the NEET projects such as:

- Include participation by departments that were not involved in the Fall 2017 pilots, so that all departments in the School of Engineering will then be involved in at least one thread.

- Each of the threads would have participation of at least one of the larger departments electrical engineering \& computer science, and, mechanical engineering.

- The thread curriculum has the potential for delaying the students' choice of major until the end of the sophomore year. 
Faculty leads for four new thread proposals presented them at the November 2017 NEET workshop titled, 'Preliminary Plans for NEET Pilot Threads that could possibly be launched in Fall 2018'. Each presentation focused on:

- Identification of the faculty lead, faculty team and participating departments.

- Description of the thread and of the new machines.

- Articulation of the high level educational goals.

- Recommendations on subjects for the sophomore year.

- Delineation of the sequence and progression of projects in the sophomore, junior and senior years.

- Plans for developing the thread community.

In January 2018, we received detailed proposals for these four new pilot threads with supporting documentation from departments. These were discussed by the core NEET faculty committee and feedback was provided to the faculty leads of each of the thread proposals. Each thread proposal was subsequently evaluated by the members of the core NEET faculty committee based on seventeen academic and organizational criteria (which were essentially the same as the criteria listed in the RFP). The unanimous recommendation was to launch two new pilot threads in fall 2018, Advanced Materials Machines (integrating aspects of materials science and engineering and mechanical engineering), and, Low Carbon Energy Systems (integrating aspects of nuclear engineering, civil and environmental engineering and mechanical engineering). These recommendations were subsequently presented to the Dean of Engineering and the Engineering Council, comprising Associate Deans and Department Heads, towards the end of February 2018.

The new threads will be formally announced to the student community early in April 2018 so that current first year students can make better-informed choices about threads and majors, before declaring their majors by the end of April 2018. The four threads available to current first year students in Fall 2018 would be: Advanced Materials Machines; Autonomous Machines; Living Machines, and; Low Carbon Energy Systems.

\section{Governance}

The core NEET faculty committee was constituted by the Dean of Engineering. It is chaired by the faculty co-leads and comprises faculty from each of the engineering departments. It follows a rigorous schedule and meets weekly to discuss, identify and implement actionable inputs on NEET including stakeholder engagement, goals, priorities, curricular and program design and organizational issues, developing the criteria for prioritizing threads, articulating the contours of what a project is in the project-centric approach, finalizing the detailed agenda for the workshops and discussing the report outs from the task groups listed below:

- Autonomous Machines thread group.

- Living Machines thread group.

- NEET Curriculum Task Group.

- NEET Projects Task Group.

- NEET Program Assessment Task Group.

- NEET Governance Task Group. 
The NEET Governance Task Group has initiated discussions to develop different organizational models as NEET moves from being a 'start-up' initiative to getting integrated across the school in the steady state.

NEET is also in the process of developing a financial model. The bulk of the cost of these efforts is associated with the costs of developing, launching and refining of a set of threads over several years. This includes:

- The recurring labor costs for lecturers, instructors and TAs to work in the project labs.

- The non-recurring labor cost to initially develop the dedicated courses and the projects.

- The capital costs of equipment for the project laboratories.

- The material and supplies cost.

- The assessment after the first delivery in 2017-18 and the second delivery in 2018-19.

- The continuing refinement and improvement of the threads over the first several years of their operation.

- A small core operations team, that helps ensures continuity and promotes institutional learning and memory; this has a recurring labor cost.

\section{What is the Feedback from Sophomores in the NEET Pilots?}

Sophomores are excited about being in NEET and appreciative of the NEET threads. They have constructive suggestions about teething problems, have come forth with interesting ideas and have conducted Q\&A sessions for the first years.

- What they like: They like being part a cross-departmental academic community, getting greater exposure to project classes, getting access to highly sought-after project classes, mentoring by NEET faculty (which is helping them to shape their paths), and that the threads are in areas that are likely to be in demand when they graduate. It is important to them that the duration of their degree will remain the same as what it would have been if they had not been in NEET.

- What could be improved: They feel that: the project experience should start in sophomore fall itself (rather than waiting till sophomore spring) and these could be through working in small groups and in vertical teams, e.g., with juniors; there needs to be greater clarity about program requirements across different majors and about roadmap options; they would like a bit more flexibility; scheduling conflicts need to be anticipated and sorted out in advance, and; having more optional lectures and workshops would make it even more interesting.

This feedback was collected anonymously through an online survey, through the NEET Seminars, and through other interactions with students. 


\section{What is the Student Community saying?}

The MIT student newspaper, The Tech, has stated that, "The impetus behind NEET ... was a combination of concerns that MIT wasn't innovating enough, and that MIT could provide much more to its students....the NEET team's goal (is) of developing curricula that do a better job at linking engineering education to application than current offerings at MIT, or indeed at any university across the world." 15

More recently, The Tech quoted Allison Lenhard '20 who is in the Autonomous Machines thread and credits it for giving her variation in her coursework. "NEET has helped me dive into a more interdisciplinary course load and tailor my MIT curriculum to suit my own career goals," Lenhard said in an interview with The Tech. She recommends any freshman to explore what NEET can offer: "I'd encourage anyone who sees a NEET thread that piques their interest to strongly consider applying, because it will definitely enhance their time at MIT," Lenhard said. ${ }^{16}$

Blogging from the Festival of Learning Expo held in January 2018, Yuliya K. '18 stated that, "NEET ... was the booth where I spent most of my time, mainly because of the contagious passion of the mentors.....Personally, I am thrilled to see this project-centric scheme, and look forward to having it implemented in my Political Science department..."17

The students have posted classes and projects in the NEET threads at courseroad.mit.edu, a student-created portal that they use extensively to map out the four-year roadmap for their majors and minors, and now, for their NEET threads.

\section{Preliminary Conclusions}

NEET is a 'start-up' in an academic setting and has definitely benefited from the focus, commitment and $24 \times 7$ energy that the start-up approach has brought to bear. This momentum needs to be supported and maintained. NEET was initiated about eighteen months ago and the pilot threads were launched last fall (Fall 2017), so though the initiative has taken big strides, it is far too early to come to meaningful conclusions for the medium to longer term.

The initial trends are encouraging. Over 5\% of the sophomore engineering population has voluntarily opted for NEET, a significantly larger number than the number of students that have typically tended to opt for many new academic programs in the past. The students are very positive and enjoy being in the threads and the student community has a favorable opinion about NEET and what it's aiming to do. There has been some attrition; one student chose to transfer to a non-engineering major that wasn't part of the thread, a couple had serious scheduling conflicts and another student is planning on medical school and that requires a set of different classes to be taken. The core group of faculty is deeply involved and NEET is starting to get a bit of traction in the faculty community; though this began as an initiative of the school of engineering other schools, e.g., the schools of architecture \& planning, humanities \& social sciences, management and science have expressed interest in working with NEET to create cross-school programs. 
There are challenges inherent in cross-departmental academic programs, e.g., scheduling conflicts. Degree audit requirements for the majors that are covered in a thread are not always aligned, since until now there was no need to. Mechanisms need to be evolved to address situations such as these.

Students need to be properly supported and guided, and good advising is even more critical; NEET and departmental faculty advisors need to coordinate to better guide the students. The role of the host department needs to be stated more explicitly, e.g., provision of space for labs and projects. It is possible in theory to defer the choice of major when there are two majors participating in a thread and it is much harder when there are three majors involved; however, this feature of NEET was not operative this year, and more data needs to be collected from the four pilots being offered this fall (Fall 2018) before we can verify this. Effective projects are hard to create or modify and require significant effort and calendar duration; full-time project staff are essential. Modules of knowledge as 'bridge' classes, could be an important role of digital learning. NEET appeals instinctively and 'sells well' to academic thought leaders, potential donors and industry. Above all, it is essential to develop, build and sustain the community of faculty, students and industry; that is the cornerstone for wider participation and sustainability.

\section{Gazing into the Future}

We would envision three or four additional threads launching in the fall of 2019 and beyond. The topics that we might pursue are in the category of general purpose technologies. In this time frame, they might include:

- Data Machines, which would build bridges between advances in data science and a wide range of problems that can benefit from such approaches.

- Smart Urban Machines, which would explore the way that cities can be strengthened and made more habitable using technology and the Internet of Things.

- Networked Machines, which would prepare students to understand networks and systems, and particularly how to control emergence in the face of complexity and ambiguity.

- Sustainable Machines, which would prepare students to understand how to create schemes for lower consumption of energy.

Figure 4 below is a visualization of how NEET and its threads could be renewed in the future. 

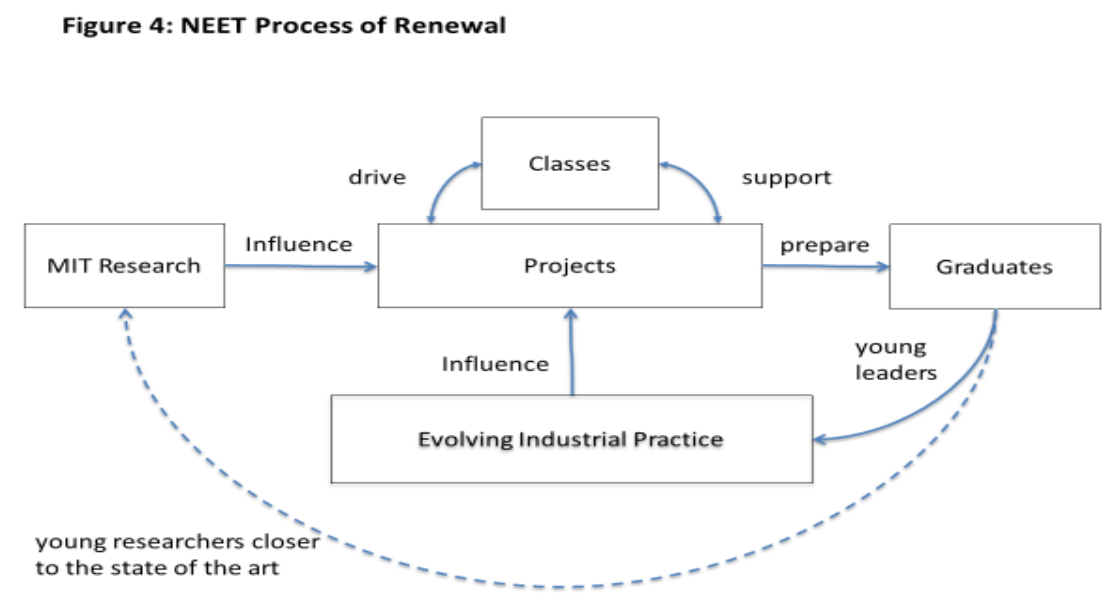

\section{References}

1. E. Crawley, J. Malmqvist, S. Ostlund, D. Brodeur and K. Edstrom, Rethinking Engineering Education. Springer, 2014.

2. S.D. Sheppard, K. Macatangay, A. Colby and W.M. Sullivan, Educating Engineers. JosseyBass, 2009.

3. D. Bok, Our Underachieving Colleges. Princeton University Press, 2006.

4. A. Johri and B.M. Olds, Editors, Cambridge Handbook of Engineering Research. Cambridge University Press, 2014.

5. S.A. Ambrose, M.W. Bridges, M. DiPietro, M.C. Lovett and M.K. Norman, How Learning Works. Jossey-Bass, 2010.

6. W.G. Tierney, Editor, The Responsive University. The Johns Hopkins University Press, 1998.

7. "Objects and Plan of an Institute of Technology," John Wilson and Son, Boston, MA,1861.

8. "Report of the Committee on Educational Survey," The Technology Press, Cambridge, MA, 1949.

9. [Gathering evidence for NEET from thought leaders, institutional and school leaders, faculty, alumni/ae, students and industry], Unpublished raw data, September 2016 - January 2017.

10. S. Mitra, 2013 TED Prize Winning Talk on "The Future of Learning”, https://www.youtube.com/watch?v=zpcEpmNbHds, 2013.

11. R. Graham, "The Global State of the Art in Engineering Education", survey report commissioned by MIT, Phase I (overview), December 2016, Phase II (deep dive into four institutions), January 2018.

12. American Association of Engineering Societies and the U.S. Department of Labor, "Engineering Competency Model," AAES website (http://www.aaes.org/model), 2015.

13. F. Kjærsdam, "Technology transfer in a globalised world: transferring between university and industry through cooperation and education", 2004 World Transactions on Engineering and Technology Education, 3(1), 63-66, 2004.

14. S.A. Male and C. Baillie, "Threshold capabilities: an emerging methodology to locate curricula thresholds," Proceedings of Research in Engineering Education Symposium, Madrid, 4th - 7th October 2011.

15. "School of engineering to pilot interdisciplinary course roads.", The Tech, August 24th, 2017. 
16. "NEET will offer two new threads this fall." The Tech, April 13th, 2018.

17. Yuliya K. MIT '18, "Festival of Learning Expo", Admissions blog, February 20th, 2018. 